\title{
Pattern formation of frictional fingers in a gravitational potential
}

\author{
Jon Alm Eriksen* \\ Department of Physics, University of Oslo, P.O. Box 1048, Blindern, 0316 Oslo, Norway \\ and Institut de Physique du Globe de Strasbourg, EOST, University of Strasbourg, CNRS, \\ 5 Rue Descartes, 67084 Strasbourg Cedex, France
}

Renaud Toussaint

Institut de Physique du Globe de Strasbourg, EOST, University of Strasbourg, CNRS, 5 Rue Descartes, 67084 Strasbourg Cedex, France and PoreLab, Department of Physics, University of Oslo, P.O. Box 1048, Blindern, 0316 Oslo, Norway

Knut Jørgen Måløy and Eirik Flekkøy

PoreLab, Department of Physics, University of Oslo, P.O. Box 1048, Blindern, 0316 Oslo, Norway

\author{
Olivier Galland \\ Physics of Geological Processes, Department of Geosciences, University of Oslo, \\ P.O. Box 1048, Blindern, 0316 Oslo, Norway
}

Bjørnar Sandnes

College of Engineering, Swansea University, Bay Campus, Fabian Way, SA1 8EN Swansea, United Kingdom

(Received 24 May 2016; published 3 January 2018)

\begin{abstract}
Aligned finger structures, with a characteristic width, emerge during the slow drainage of a liquid-granular mixture in a tilted Hele-Shaw cell. A transition from vertical to horizontal alignment of the finger structures is observed as the tilting angle and the granular density are varied. An analytical model is presented, demonstrating that the alignment properties are the result of the competition between fluctuating granular stresses and the hydrostatic pressure. The dynamics is reproduced in simulations. We also show how the system explains patterns observed in nature, created during the early stages of a dike formation.
\end{abstract}

DOI: 10.1103/PhysRevFluids.3.013801

\section{INTRODUCTION}

Subsurface flows tend to converge on high-conductivity pathways such as rock fractures, joints, and faults. Flow of oil and gas in fractured reservoirs, groundwater transport, magma flow, and pollutant transport in fractured porous media is therefore often dominated by the interactions between the flowing fluids, the confining geometries, and granular rock fragments residing in the cracks or faults.

A range of flow patterns can emerge when one fluid displaces another fluid in such confined spaces $[1,2]$. These flow patterns are caused by the interplay between different stabilizing and destabilizing effects, such as surface tension, gravity, pore size fluctuations, wettability properties, and granular effects. Viscous fingering is a well-known example of a fluid flow instability. An initially straight interface between two immiscible fluids of different viscosities develops undulations that grow to form fingers when the less viscous fluid invades the more viscous host fluid [3-6]. In rough fractures

\footnotetext{
*jonaerik@fys.uio.no
} 
or a porous medium, disorder in the form of variations in pore sizes perturbs the invading interface, generating fractal two-phase flow structures with no intrinsic length scale [7-11].

Gravity has a profound effect on the flow patterning in situations where a density difference between the fluids exists and where the flow geometry is not strictly horizontal. For example, in density-driven convection, the interface between a dense fluid overlying a less dense fluid becomes unstable, with dense fluid fingers sinking and low-density fingers rising [12-14]. With the less dense fluid on top, on the other hand, the hydrostatic pressure stabilizes the interface at a given height. During slow drainage of a porous medium, a competition exists between the stabilizing effect of gravity and the pore scale disorder that increases the roughness of the invasion front $[15,16]$.

Rock fractures and other high-permeability flow paths can be filled with granular debris and fault gouge from cataclastic processes and erosion $[17,18]$, materials carried by fluid flow, or, in the case of magma flow, crystal particles precipitated from the melt [19-21]. Multiphase flows involving both a combination of different fluids and a loose packing of granular materials have proved a particularly rich vein of pattern formation as frictional fluid dynamics is added to the well-known two-phase flow mechanisms [22]. Recently observed flow patterning processes include multiphase fracturing of deformable granular packings [22-27], decompaction fingers [28], frictional fingers, and bubbles $[22,29,30]$.

Here we introduce gravity as a new parameter in experiments where air displaces a liquidgranular mixture during drainage of a Hele-Shaw cell, by imposing shallow tilt angles. The receding interface accumulates a front of granular material, and an instability caused by a competition between surface tension and frictional forces results in an emerging pattern of frictional fingers, canals of air separated by branches of compacted grains, as also observed in horizontal systems $[22,29,31]$. In the case of the tilted system, the symmetry breaking by gravity changes the pattern formation dynamics by stabilizing the drainage front, resulting in alignment of the finger structures. We further expand a frictional finger simulation model [32] to include hydrostatic pressure and develop a theoretical prediction for transitions in the pattern formation dynamics.

We find that the key to the finger alignment direction is a competition between gravity and fluctuations of the intergranular stresses. Analogous to drainage in porous media [15], random fluctuations in threshold pressures cause a disruption of the stabilizing effect of gravity. However, unlike porous media, there is in our system a spontaneous emergence of a characteristic length, the finger width $2 \Lambda$ ( $\Lambda$ denotes here half the finger width). The magnitude of the disruption of the invasion front becomes a relative quantity with respect to this length scale. We show that the basic assumption that the effective granular friction stresses at the interface arises as a sum of a set of uncorrelated random contributions is sufficient to give a theoretical prediction of the transition between the different pattern morphologies.

We also show how the pattern forming mechanism provides further understanding of the smallscale flow properties during magmatic dike formations, i.e., the penetration of a sheet of magma into a fracture of a preexisting rock body [33-35]. The small-scale flow properties during this formation, when magma interacts with the host rock, is largely unknown [36], as the formation occurs deep beneath the earth's crust. Rock faces in the Israeli desert [37,38] display aligned finger structures which were formed during a dike formation. The structures have previously been attributed to viscous fingers, due to the Saffman-Taylor instability [3], between the fluidized host rock and a less viscous dike-related fluid in front of the invading magma [38]. We hypothesize here that intergranular frictional forces between quartz grains in the fluidized host rock and not viscous forces of the fluids govern the formation of the pattern.

\section{EXPERIMENT}

Consider a rectangular $200 \times 300 \mathrm{~mm}^{2}$ Hele-Shaw cell with a gap spacing $h=0.5 \mathrm{~mm}$ [Fig. 1 (a)]. The cell is sealed along the sides and base; the upper end is open to the ambient air. In preparation for the experiment, a granular material suspended in a 50\% (by volume) water-glycerol 


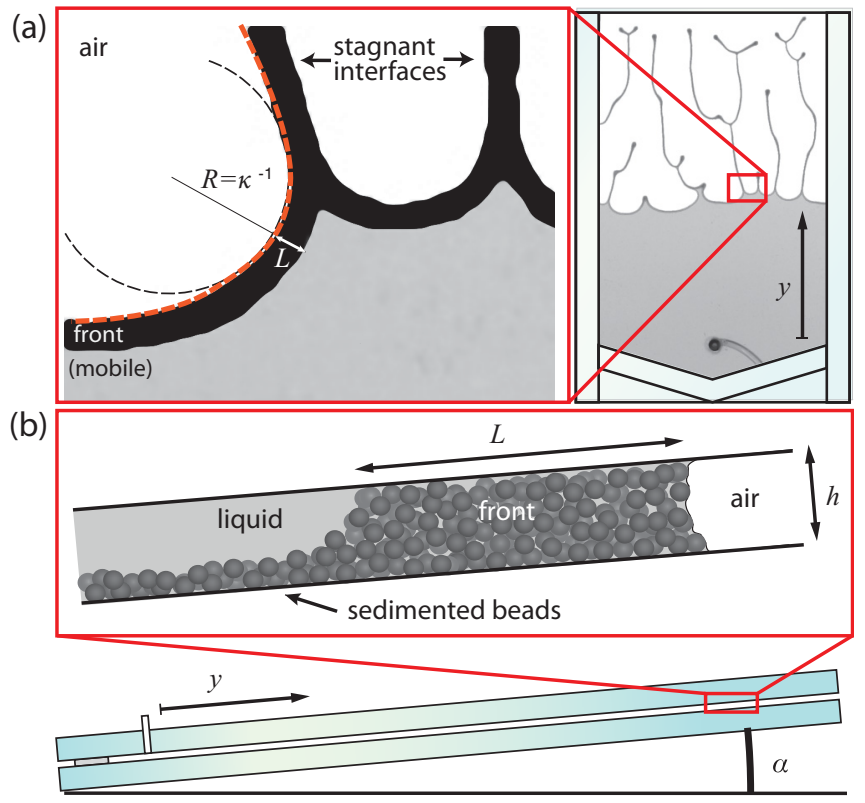

FIG. 1. (a) Top view of the Hele-Shaw cell. The coordinate $y$ is running from the outlet towards the upper edge of the cell; $\kappa$ is the curvature (inverse of the in-plane radius of curvature $R$ ) along the interface (orange dashed line). The front is a region of accumulated grains along the air-liquid interface; $L$ is the thickness of this front. The cell is $20 \times 30 \mathrm{~cm}^{2}$. (b) Side view. The cell is tilted by an angle $\alpha$. The cell gap is $h=0.5 \mathrm{~mm}$. The filling fraction $\phi$ is the height of the initial sedimented granular layer relative to $h$.

mixture is injected into the horizontal cell through an inlet-outlet hole close to the base of the cell. Excess mixture spills through the open edge such that the granular suspension fills the entire cell. The granular material, spherical glass beads with mean diameter $80 \pm 10 \mu \mathrm{m}$, settles out of suspension, forming a layer of grains resting on the lower glass plate of the cell. The height of this layer, relative to the cell gap, is denoted by $\phi$ and quantifies the initial filling fraction of the injected granular mixture relative to the random loose-packing fraction of the grains. The glass beads are polydisperse and the variation in size prevents crystallization of the sedimented bead packing. The densities of the glass beads and the water-glycerol mixture are $\rho_{g}=2.4 \mathrm{~g} / \mathrm{cm}^{3}$ and $\rho=1.13 \mathrm{~g} / \mathrm{cm}^{3}$, respectively. The bead-fluid density contrast makes the beads sediment on the bottom plate. We note that the invasion process described in this paper is limited to noncolloidal, sedimenting granular material, with the capillary length of the interface as the upper limit in terms of grain size. The gap to grain size ratio is approximately 6:1 in the experiments presented here and we do not expect that the results are transferable to monolayered systems.

The long side of the cell is tilted by an angle $\alpha$ relative to the horizontal plane [Fig. 1(b)]. Here we report only results for shallow tilt angles $\left(0^{\circ} \leqslant \alpha \leqslant 5^{\circ}\right)$ where no sliding of the granular layer takes place. The experiment commences by slowly draining fluid from the outlet at the base at constant withdrawal rate $q=0.07 \mathrm{ml} / \mathrm{min}$ unless otherwise noted, using a syringe pump (WPI, Aladdin 1000). The value for the withdrawal rate is chosen such that the experiment resides well within the frictional stick-slip regime [22], where the dynamics is independent over a wide range of withdrawal rates. The withdrawal rate is slow enough to leave the layer of grains resting on the bottom plate undisturbed by the fluid flow. As fluid is slowly drained, air starts to invade the cell from the open elevated edge. The meniscus bulldozes the loose granular material ahead of it and does not penetrate the pore space between grains. The system is imaged 

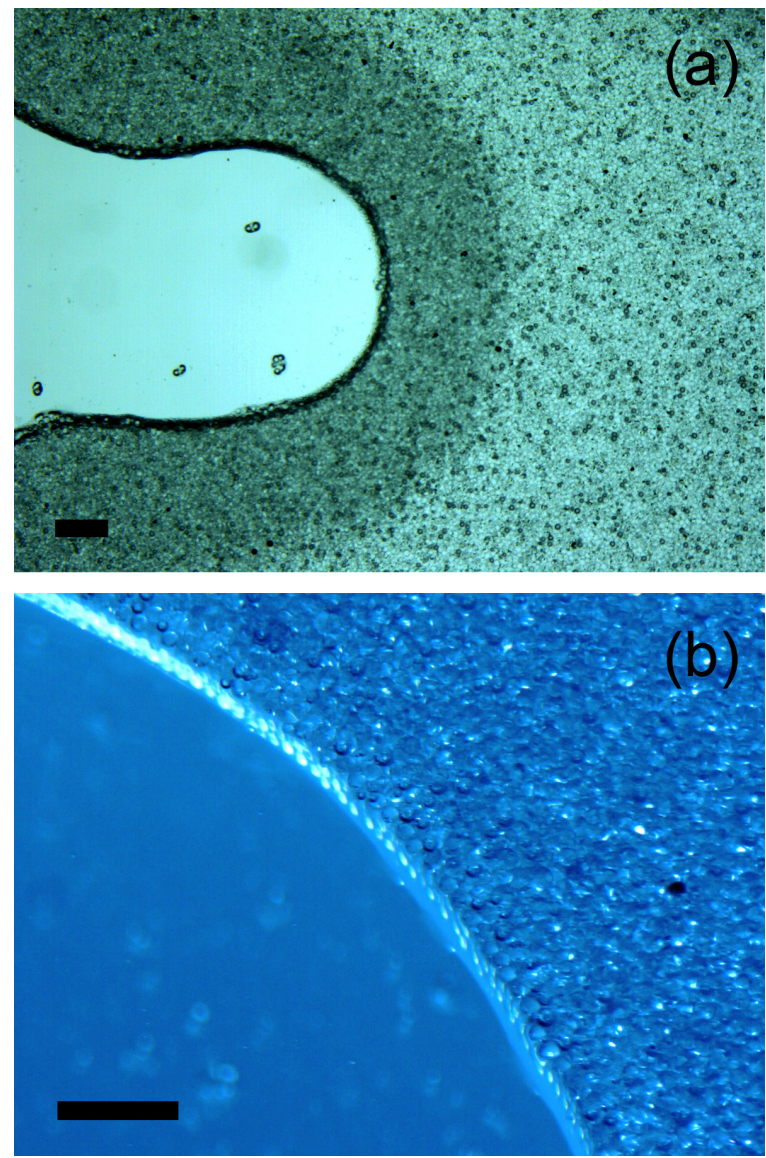

FIG. 2. (a) Air finger displacing submerged grains. The air-liquid-grain interface appears as a black line. The interface is surrounded by a compaction front, which appears darker than the undisturbed sedimented layer of grains to the right in the image. The scale bar is $1.0 \mathrm{~mm}$. (b) Close-up of the interface showing grains in contact with the interface as a bright band due to reflection of illumination from the side. The scale bar is $0.5 \mathrm{~mm}$; $q=0.07 \mathrm{ml} / \mathrm{min}, \phi=0.4$, and $\alpha=0$ for both images.

from underneath using a PL-B742U Pixelink camera and illuminated by a white screen placed above.

As the air displaces the liquid-grain mixture, the air-liquid interface sweeps the grains along and granular material accumulates ahead of the invading interface. The granular accumulation front forms a dense packing that fills the gap between the two confining plates of the Hele-Shaw cell (see Figs. 1 and 2). Only a small section of the interface moves at any given time and the motion consists of incremental displacements, as the air fills an ever-increasing volume. A moving section tends to continue its motion over many consecutive increments before it stops and the motion continues at another section. The interface develops frictional fingers of air surrounded by a front [22,29,31], with a characteristic finger width. When different fingers move towards each other, their fronts combine and their interfaces stagnate. The evolution continues until either the whole cell is filled with air and stagnant fronts or the air reaches the outlet.

When the cell is fixed horizontally $\left(\alpha=0^{\circ}\right)$, the finger directions are disordered and isotropic and the resulting patterns are labyrinth structures of stagnant fronts [29,31]. When the cell is tilted, the frictional fingers are observed to align. The direction of alignment changes as we vary $\alpha$ or $\phi$. 


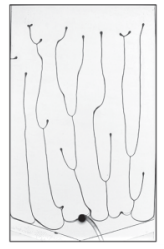

$\phi=0.025$

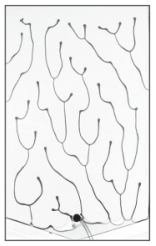

0.05

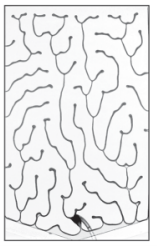

0.1

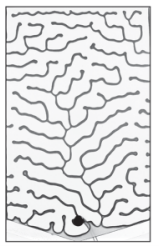

0.2

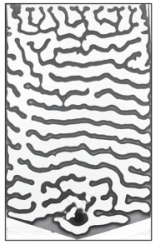

0.4

FIG. 3. Final configuration of the experimentally observed pattern at constant tilt angle $\alpha=4^{\circ}$ with varying filling fraction $\phi$. Residual compacted granular material appears dark and empty regions of the cell appear white. The finger alignment changes direction from vertical to horizontal as $\phi$ increases. Each image frame is $200 \mathrm{~mm}$ wide.

Figure 3 shows the residual patterns of granular material in the shape of narrow branches after all the grains have been packed at the end of each experiment. The figure displays results from a series of experiments with increasing filling fraction, with the tilt angle kept constant at $\alpha=4^{\circ}$. The pattern of residual granular material bears witness to the dynamics of the invasion process. At low $\phi$, the air fingers march downward, from top to bottom, leaving granular branches aligned with the direction of gravity (vertical in the images). At high $\phi$, the system makes a transition to sideways growing air fingers, leaving a trail of horizontally aligned granular branches. Alternatively, by keeping $\phi$ constant and increasing the tilt angle, it is possible to go from random labyrinthine pattern to horizontal alignment and then to vertical alignment at high $\alpha$. Figure 4 shows the residual patterns imaged at the time of air breakthrough at the outlet for $\alpha=0^{\circ}, 2^{\circ}$, and $4^{\circ}$. Note the incomplete drainage of the flat cell $\alpha=0^{\circ}$. The absence of the height stabilization allows the air fingers to invade in random directions, leaving pockets of grain-liquid mixture undisturbed behind the actively growing fingers.

In the low- $\phi$ to high- $\alpha$ range, hydrostatic height stabilization of the receding interface dominates the dynamics and the fingers advance side-by-side downward, parallel to the gravitational field along the cell [Fig. 5(a), Supplemental Material (SM) video 1 [39]]. Lateral growth is inhibited by the presence of neighboring fingers on both sides; each finger is confined to downward growth. A finger will terminate its movement if it is bypassed and sealed off by its neighboring fingers. A finger can also split in two if a small region along the finger tip gets stuck, and each side of this region evolves to separate fingers. This typically happens when a finger tip widens, which seems to happen in conjunction with the termination of a neighboring finger. Finger termination and tip splitting occur at approximately equal frequencies [see Fig. 5(a)], maintaining a steady-state evolution of the experiment and a constant mean finger width. We note that these patterns looks remarkably similar to patterns generated when simulating retraction of a dewetting suspensions [40], although the setup is completely different.
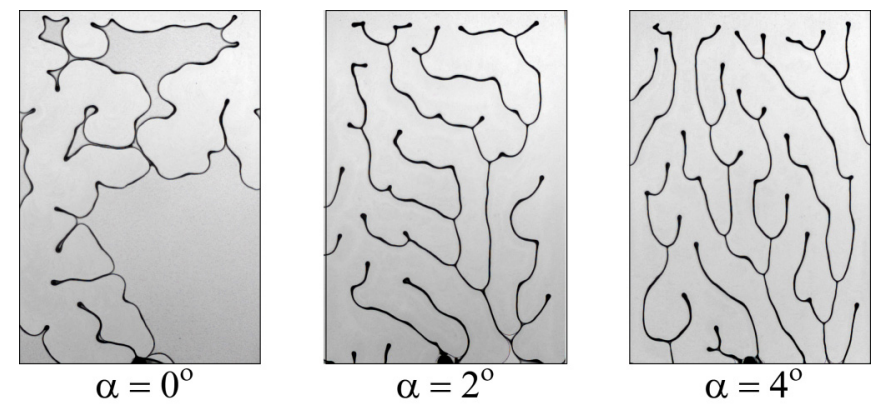

FIG. 4. Final configuration of experimental pattern at different tilt angles $\alpha$ at filling fraction $\phi=0.05$. Each image frame is $200 \mathrm{~mm}$ wide. 


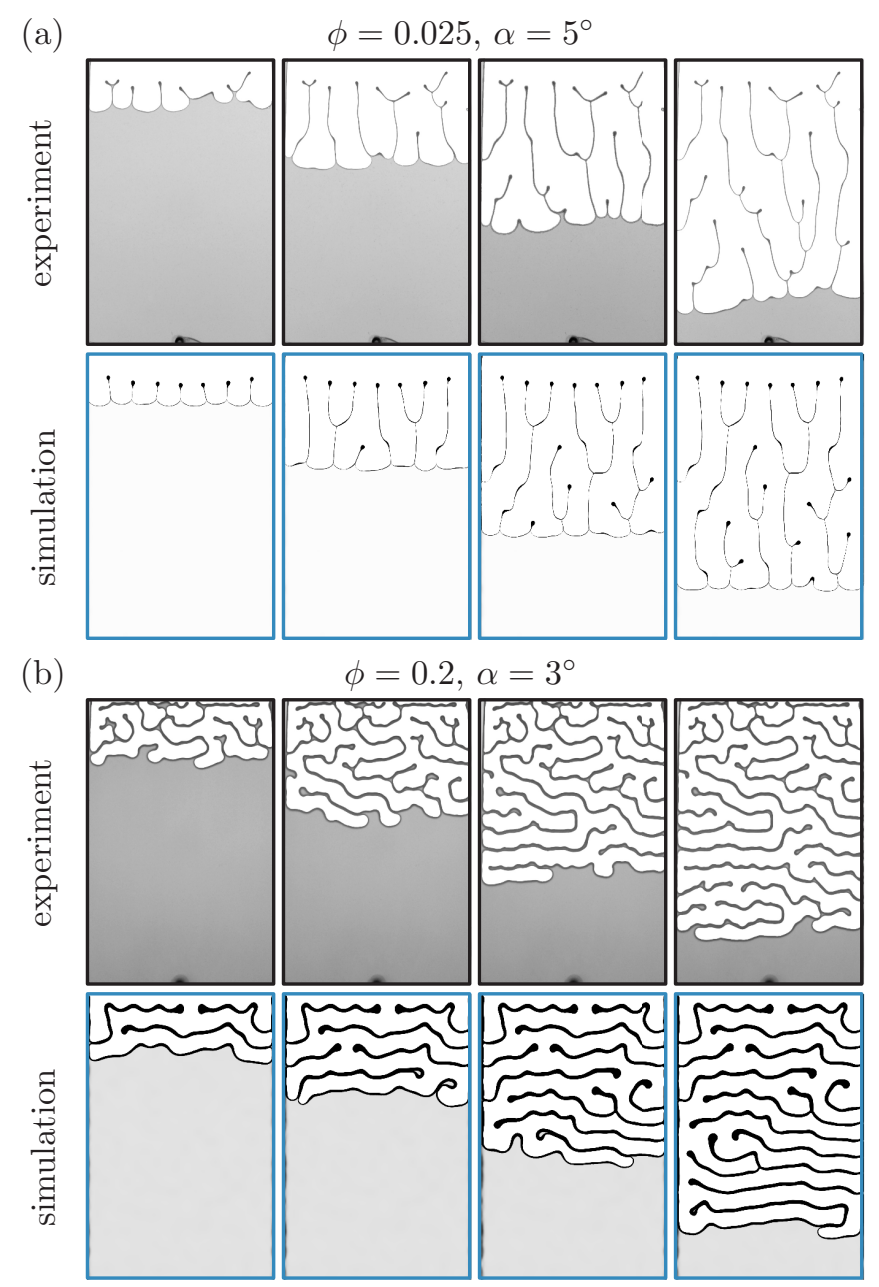

FIG. 5. Snapshots of the dynamics, experiments versus simulations. (a) The pattern is dominated by vertically aligned fingers at $\phi=0.025$ and $\alpha=5^{\circ}$. (b) The pattern is dominated by horizontally aligned fingers at $\phi=0.2$ and $\alpha=3^{\circ}$. See (a) SM video 1 and (b) SM video 2 [39].

As we increase $\phi$ and reduce $\alpha$, we observe a gradual transition in the alignment; the fingers tend to grow with a directional component transverse to the hydrostatic pressure gradient. In the intermediate range of $\phi$ and $\alpha$, hydrostatic stabilization of the front occurs, but local pressure fluctuations enables some fingers to get ahead. Sideways growth is preferred for a finger that extends beyond its neighbors due to the hydrostatic pressure gradient. The finger which manages to get ahead fills a larger fraction of the horizontal direction and advances layer by layer, creating a pattern of horizontal lines [Fig. 5(b), SM video 2 [39]]. In the high- $\phi$ to low- $\alpha$ range the local pressure fluctuations dominate over the stabilizing effects and alignment is lost. A phase diagram of the alignment behavior of the end configurations is shown in Fig. 6.

\section{MODEL}

As the dynamics are manifested by incremental movements of confined regions of the interface, it is reasonable to assign a yield pressure threshold to every point along the interface. When the pressure difference at the interface exceeds the threshold at the weakest point along the interface, the 


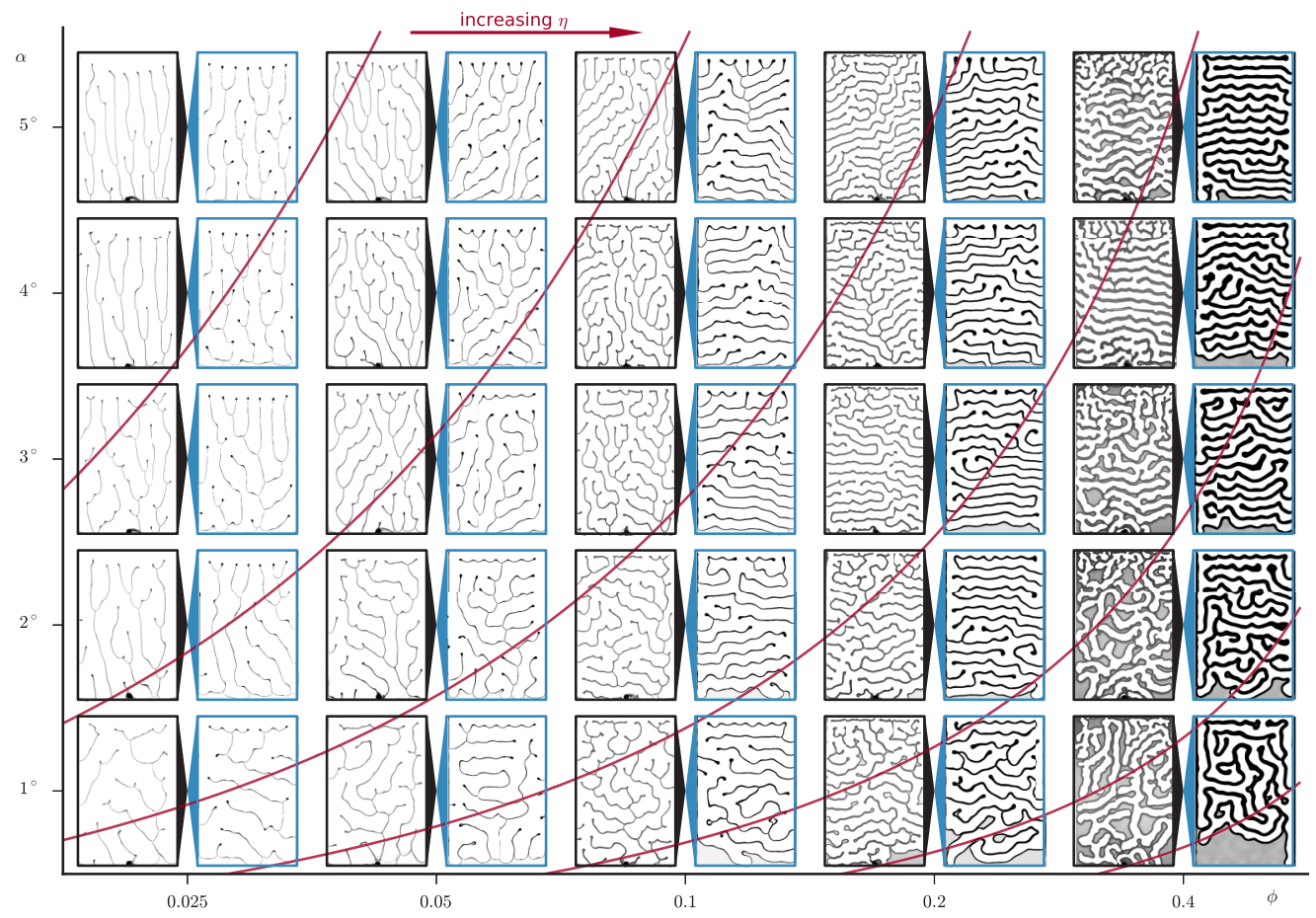

FIG. 6. Pairwise comparison of the final configuration of experiments (black left frames) to simulations (blue right frames) for different values of the filling fraction $\phi$ and the tilting angle $\alpha$. The red lines indicate contours of constant $\eta$, which are estimated up to a constant factor in Eq. (9). As $\eta$ increases, the vertical alignment turns into horizontal alignment and then into no alignment. The value of $\eta$ doubles for every contour. The gravitational pull is pointing downward in every frame.

interface locally to that point deforms and moves a small step towards the liquid phase. This approach has successfully modeled labyrinth patterns in a similar setup [29,31], but without considering the hydrostatic pressure differences induced by the tilting of the cell. In order to quantify the yield pressure threshold, we will assign two local parameters to the interface: the front thickness $L$ and the apparent in-plane curvature $\kappa$.

The front thickness $L$ is the distance from the air-liquid interface, in the perpendicular direction, to the region of the liquid mixture where the beads no longer fills the whole cell gap (see Fig. 1). Note that the packing of beads in the front remains in a static configuration before a potential movement. We assign a yield stress $\sigma_{Y}(L)$ to every point along the interface, which captures the static frictional properties of the front. To be precise, $\sigma_{Y}$ is the yield stress acting normal to the plane which approximates the air-liquid interface. This yield stress has previously, in the context of labyrinth patterns [29] and of plug formations in narrow tubes [41], been assumed to be exponentially increasing in front thickness $L$. The exponential behavior can be justified by considering Janssen's model for stresses in packings of grains, which assumes a linear relationship between the principal stresses in the packing, in conjunction with the static Coulomb frictional stresses at the plate boundaries of the cell. The yield stress may also have a curvature dependence, as described in Ref. [30]. In the following, we will, however, describe the yield stress as a linear function in $L$,

$$
\sigma_{Y}(L)=\frac{\sigma_{\xi}}{\xi} L
$$

for simplicity. The numerical comparison to the experimental behavior in the subsequent section will validate this approximation as sufficient for the range of parameters that we consider here. 
The expression in Eq. (1) has two interpretations. We can interpret it as a linearization of a more complicated function of $L$, e.g., the exponential behavior assumed in [29,41]. In this case, the length parameter $\xi$ should equal the cell spacing $h$, up to geometric factors $\simeq 1$ [consider the Taylor expansion of Eq. (12) in Ref. [29]]. Alternatively, we can interpret the yield stress as a sum of consecutive force bearing arc chains [32] which transmit frictional stresses $\sigma_{\xi}$ from the cell plates to the beads at the air-liquid interface. The characteristic length of these chains is $\xi$ and the total number of chains scales with the size of the front and therefore linearly in $L$. It is reasonable to assume that $\xi \simeq h$, also in this approximation, due to the confined geometry.

The air-liquid surface tension at the interface acts at two different scales. At the small scale, the interface makes bridges between wetting beads. Each point on a meniscus can be characterized by two principal radii of curvature. By the Young-Laplace equation, the pressure drop over a meniscus is proportional to the mean of the principal curvatures. This means that in a static configuration, each meniscus has the exact same mean curvature, up to differences in the hydrostatic liquid potential, which we can ignore in a horizontally oriented cell.

At a larger scale, we can identify a curvature which is averaged over several neighboring beads. For our Hele-Shaw setup, the principle directions of the average curvature are the in-plane and the out-of-plane directions with respect to the cell plane. We will disregard the curvature component in the out-of-plane direction of the cell, i.e., the curvature of the interface as it is illustrated in the cross section in Fig. 1(b). The out-of-plane curvature is supposed roughly constant, i.e., the surface stress related to this component is constant along the in-plane direction of the interface and does, at our level of description, only contribute to a constant global pressure drop. It plays no role when we later need to determine the minimal yield stress.

The large-scale surface behavior, i.e., the surface behavior averaged over many neighboring interbead menisci, can be characterized by an effective surface tension $\gamma$ [29]. The effective tension acts against the increase of the apparent interface area during the displacement process and the associated pressure difference is simply $\gamma \kappa$.

We can now quantify the local yield pressure threshold. Let $\Delta p$ be the difference between the air pressure $p_{\text {air }}$, which is considered constant, and the liquid pressure at the outlet of the cell $p_{\text {outlet }}$. We assume that a section of the interface is mobilized if

$$
\Delta p \geqslant \gamma \kappa+\frac{\sigma_{\xi}}{\xi} L-y \rho g \sin \alpha
$$

The first and second terms on the right-hand side are the effective surface stress and the yield stress [Eq. (1)] described above. The last remaining term is the hydrostatic pressure relative to the base of the cell, $y$ is a coordinate running along the cell from the outlet, $g$ is the gravitational acceleration, and $\rho$ is the liquid density. This amounts to saying that the local pressure in the fluid behind the meniscus $p_{\text {air }}-\gamma \kappa$ is equal to the sum of the solid and the fluid stress there. The fluid stress there is $p_{\text {outlet }}-y \rho g \sin \alpha$, hence the solid stress there is $\sigma_{\perp \text { solid }}=p_{\text {air }}-\left(p_{\text {outlet }}-y \rho g \sin \alpha\right)-\gamma \kappa$. If the solid stress is equal to or larger than $\sigma_{\xi} / \xi L$, the grain pack slides locally. The pressure difference $\Delta p$ will increase when the whole interface remains static and liquid is drained from the system. The next moving section, at any given time, is identified by local parameters $\kappa, L$, and $y$, which minimizes the right-hand side of Eq. (2). As the section yields and moves a small step towards the liquid, the local parameters are changed due to the deformation and the accumulation of new beads onto the front.

\section{A. Numerical validation}

We can reproduce the experimental behavior in a numerical simulation. The numerical scheme has previously been used to simulate finger behavior in a flat cell [32]. We present here a summary of the numerical strategy and the modifications which are needed for the tilting of the cell. Further details of the numerical scheme are described in Ref. [32].

The fluid interface (i.e., the boundary of the gas phase) can be represented as a chain of nodes, labeled by an index $i$, where each node carries information of the spatial coordinates $\left(x_{i}, y_{i}\right)$ and its nearest neighbors $i \pm 1$. Such a chain can conveniently be implemented like a doubly linked 
list. We couple this chain of nodes to a two-dimensional mass field, representing the grains. The complete filling of the cell gap, i.e., the region which constitutes the front, is indicated by the region of the mass field which exceeds a threshold value. We make sure that the region of the mass field adjacent to the chain, i.e., the region of the front, exceeds this threshold in the initial configuration of the system. The imposed dynamics described below will maintain this state.

For each node we can identify the two local properties. First, the local front length $L_{i}$ is represented as the shortest distance from any given node to a cell in the mass field which takes a value below the threshold. This cell will be referred to as the link cell associated to the node. Second, we can approximate the local curvature $\kappa_{i}$ at node $i$ by numerical differentiation of a spline approximation of the nearest and next-nearest neighbors $\{i, i \pm 1, i \pm 2\}$. By discretizing the right-hand side of Eq. (2), we can now identify a pressure threshold $T_{i}$ for each node,

$$
T_{i}=\gamma \kappa_{i}+\frac{\sigma_{\xi}}{\xi} L_{i}-y_{i} \rho g \sin \alpha
$$

The dynamics of the system is generated by iteratively moving the node with the minimal value of $T_{i}$, an infinitesimal distance towards the fluid phase, in the perpendicular direction to the interface. At each step we need to accumulate new beads from the initial distribution to the front. This can be achieved by adding the gathered bead mass which corresponds to the infinitesimal displacement to the link cell of the node. If this cell reaches the threshold value, a new link cell will be assigned and the rest mass will be distributed there. This approach will make sure that the bead mass field is conserved. The chain is interpolated with new nodes as the interface grows, keeping the resolution of the representation of the interface constant, and the local quantities $\kappa_{i}$ and $L_{i}$ are recalculated in a neighborhood along the chain near the moving node.

Note that there is no time in this numerical approach. We can, however, estimate the time from the volume of the air phase, as we know that the drainage rate $q$ is constant. This allows us to compare the experimental results to the numerical simulation during the evolution of the patterns. The dynamics is deterministic and the random behavior is a result of perturbed initial conditions and imposed quenched fluctuations in the initial mass field. Note that the random fluctuations in the mass field will induce fluctuations in $L_{i}$, as mass is accumulated. These fluctuations scale with $\sqrt{L_{i}}$ as $L_{i}$ corresponds to a sum of multiple randomly distributed masses. This effectively induces fluctuations in $T_{i}$ evaluated at each node.

We use $\sigma_{\xi} / \xi=16 \mathrm{kPa} / \mathrm{m}$, which is an estimate based on comparison between experimental results and the theoretical expression for finger width [32]. For the effective surface tension we use $\gamma=60 \mathrm{mN} / \mathrm{m}$ [29]. The similarity between the simulated and experimentally observed patterns (see Fig. 6 and SM videos 1 and 2 [39]) validates our theoretical understanding. A noticeable difference between simulations and experiments is that the experiments terminate once the interface reaches the outlet, which can result in incomplete drainage and fluid pockets left behind the advancing front (see, e.g., $\alpha=0$ in Fig. 4 and the high- $\phi$ to low- $\alpha$ results in Fig. 6). The simulations run until the interface reaches the bottom boundary.

\section{B. Transition of alignment direction}

To understand the transition between horizontally and vertically oriented finger behavior, we need first to quantify the variations in the yield pressure threshold [Eq. (2)]. It is hard to quantify the exact numerical value of these variations, but it will suffice for our purposes to determine how the variance scales with $L$. We argue that the simplest and most plausible scaling is that the variance is linear in $L$, i.e., $\operatorname{Var}\left(\sigma_{Y}\right) \propto L$, such that the standard deviation of $\sigma_{Y}$ is proportional to $\sqrt{L}$. A sum of $n$ uncorrelated and identically distributed variates exhibit a linear scaling in $n$. It is reasonable to assume that the yield stress $\sigma_{Y}$ also arises as the sum of uncorrelated contributions. If we interpret Eq. (1) to be a sum of force bearing arc chains of length $\xi$, each of which contributes with a varying yield stress with a mean value of $\sigma_{\xi}$, then the total variation will scale with the number of these chains. 
As the number of chains scales with the size of the front, we have that $\operatorname{Var}\left(\sigma_{Y}\right) \propto L$. Alternatively, the variations in $\sigma_{Y}$ might be induced by variations in front thickness $L$ itself. The front length $L$ is a result of the accumulation of beads after many small incremental steps. Heterogeneity in the initial packing fraction will therefore induce a variance in $L$ which also is proportional to $L$ itself. Note that in the numerical simulations, this is indeed how we induce the fluctuations in $\sigma_{Y}$. The value of $\sigma_{\xi}$ is kept fixed (it is not a random variable) and the fluctuation are imposed in the initial bead field.

We can compare these variations to the hydrostatic pressure difference over a horizontally oriented finger. The finger width is $2 \Lambda$ and the corresponding hydrostatic difference is $2 \Lambda g \rho \sin \alpha$. The ratio between the standard deviation of the yield stress and the hydrostatic difference of a horizontally oriented finger is therefore

$$
\eta=\frac{\left[\operatorname{Var}\left(\sigma_{Y}\right)\right]^{1 / 2}}{2 \Lambda g \rho \sin \alpha} \propto \frac{\sqrt{L}}{\Lambda \sin \alpha} .
$$

The last expression, which is proportional to $\eta$, contains all the terms which implicitly depend on $\alpha$ and $\phi$. This expression indicates the behavior of the alignment, up to an unknown constant. When the contribution of stress fluctuations is comparable to the stabilizing pressure $(\eta \simeq 1)$ a finger can get ahead of its neighbors and grow sideways, orthogonal to the direction of gravity. For $\eta<1$, the fluctuations fail to disrupt the side-by-side finger growth. For $\eta>1$, the fluctuations dominate over the stabilizing effect and the alignment is lost. We can only estimate $\eta$ up to a multiplicative constant, as the numerical value of the stress variations of $\sigma_{Y}$ is hard to identify. This will, however, suffice for identifying the contour lines in the $(\alpha, \phi)$ plane, which have similar alignment properties. To identify these contour lines, we first need to express $\Lambda$ and $L$ in terms of $\phi$.

Let $A$ and $C$ be, respectively, the area and the circumference of the air phase, as seen from above, and let $h$ be the cell gap. The pattern is dominated by finger structures such that $A=C \Lambda$. We assume that $L$ is approximately constant along the interface such that $C L$ is the total area of the front. Mass conservation gives that $h(C L+A) \phi=h C L$, which under the substitution $\Lambda=A / C$ implies that

$$
L=\Lambda \frac{\phi}{1-\phi}
$$

A more detailed derivation, which differentiates between the front thickness at the sides and the tip of the fingers, yields correction terms to this expression (see Ref. [32]).

The work of a typical displacement $\delta w$ has two contributions when we set $\alpha=0$ for simplicity. First, the stretching of the interface contributes with $\gamma h \delta C$, where $\delta C=\delta A / \Lambda$, which follows from the assumption of constant $\Lambda$. Second, there is the work done against the granular stresses $\sigma$ in the front. This work has a slightly more complicated origin as it depends on variations in the front thickness, as described in Ref. [32]. At the fingertip where most of the displacement takes place, the curvature $\kappa$ and the corresponding surface tension forces are largest, while the front thickness and friction will be smallest. Away from the fingertip the front thickness increases to its final value $L$.

However, for the present purposes of estimating $\eta$, we will be content with the leading-order behavior of this work, and this follows from the simplifying assumption that the frictional value is done against a front thickness of constant value $L$. Then the work is simply given as $\operatorname{sh} \delta x \sigma$, where $s$ is the typical width of a moving segment and $\delta x$ is the distance the interface advances. Since all forces act normal to the interface we have that $s \delta x=\delta A$. Finally, we can approximate $\sigma$ by the yield stress $\sigma_{Y}$ [Eq. (1)]. Putting the terms together and dividing by the displacement duration gives the work rate

$$
\frac{\delta w}{\delta t}=\left(\frac{\gamma}{\Lambda}+L \frac{\sigma_{\xi}}{\xi}\right) h \frac{\delta A}{\delta t},
$$


where $h \delta A / \delta t$ equals the constant compression rate when averaged over many stick-slip events. Substituting Eq. (5) and minimizing Eq. (6) with respect to $\Lambda$ gives

$$
0=\frac{d}{d \Lambda} \frac{\delta w}{\delta t} \Rightarrow 0=\frac{d}{d \Lambda}\left(\frac{\gamma}{\Lambda}+\Lambda \frac{\phi}{1-\phi} \frac{\sigma_{\xi}}{\xi}\right)
$$

which corresponds to the assumption that the pattern evolves in a way that minimizes the work. Note that the assumption of minimization of work is equivalent to minimization of the yield stress threshold at the finger tip [29,32]. This implies

$$
\Lambda=\sqrt{\frac{\gamma \xi}{\sigma_{\xi}} \frac{1-\phi}{\phi}} \propto \sqrt{\frac{1-\phi}{\phi}} .
$$

This expression differs from the one given in Eq. (15) of Ref. [32], as the inclusion of $L$ variations along the front produces a $\phi$-dependent prefactor to the effective surface tension in Eq. (6), which is absent here. However, the expressions agree to leading order in $\phi /(1-\phi)$, which will suffice for our present purposes. Note that the model predicts a $\Lambda \propto \sqrt{h}$ dependence on cell gap (assuming $\xi \simeq h$, which is consistent with previously obtained experimental and numerical results for varying gap height in horizontal systems [29,31].

We can now use Eqs. (5) and (8) to rewrite Eq. (4) as a function of $\phi$ and $\alpha$,

$$
\eta \propto \frac{1}{\sin \alpha}\left(\frac{\phi}{1-\phi}\right)^{3 / 4} .
$$

Indeed, contours of constant $\eta$ correspond to equal qualitative alignment behavior, as shown in Fig. 6.

To better quantify the transition of the finger direction, we investigate the statistical properties of the air-grain interface in the patterns presented in Fig. 6. Standard image processing techniques allow us to identify the contour paths of the air-liquid interface. To get a statistical measure of the direction of the finger structures, we investigate the properties of a random sample of tangents associated with points along these contours. The contour paths are smoothed at a length scale which corresponds to $1 \mathrm{~mm}$ for the experimental images such that variations due to the presence of grains are ignored. Moreover, we restrict our sample to only include tangents from points along the contours which are at least $1 \mathrm{~cm}$ from the cell boundaries, i.e., we disregard a margin corresponding to $5 \%$ of the cell width. This is done to remove fingers which grow along the boundaries from the tangent sample. With each tangent, we can associate an angle $\theta$ which is defined to be the smallest angle between the tangent line and any line parallel to the length direction of the cell, i.e., parallel to the average flow. Therefore, $\theta$ is an acute angle in the interval $\theta \in[0, \pi / 2]$, which takes the value $\theta=0$ for points along the interface of fingers which grow parallel to the gravitational field along the cell and $\theta=\pi / 2$ for fingers which grow in the perpendicular direction. Figure 7 shows histograms corresponding to samples from each of the patterns in Fig. 6, for both the experimental and simulated results. We see that the histograms corresponding to parameters $(\alpha$ and $\phi)$ for which the system is dominated by vertical fingers are skewed towards $\theta=0$ (e.g., for $\alpha=5^{\circ}$ and $\phi=0.025$ ). In contrast, the histograms corresponding to parameters for which the system is dominated by horizontal fingers are skewed towards $\theta=\pi / 2$ (e.g., for $\alpha=5^{\circ}$ and $\phi=0.4$ ).

To better illustrate the alignment transition, we consider two properties of each sample: first, the median, i.e., the value which separates the larger half from the smaller half of the sample, and second, the smallest interval of the $\theta$ sample, which contains a fixed proportion $p$ of the sample data. We will denote this interval by $\operatorname{SI}(p)$. While the median serves as a measure of the center of the underlying distribution, $\operatorname{SI}(p)$ indicates an interval with a high probability in the underlying distribution. If the underlying distribution is unimodal, the mode (i.e., the maximum) of the distribution will be contained in this interval.

We plot the median and $\operatorname{SI}(p)$ with $p=0.3$ for each sample against $\ln \eta$ in Fig. 8. As $\eta$ increases we see the transition from vertically to horizontally aligned fingers, i.e., from $\theta=0$ to $\theta=\pi / 2$. Note that $\operatorname{SI}(p)$ captures this transition better than the median, as the underlying distribution seems 


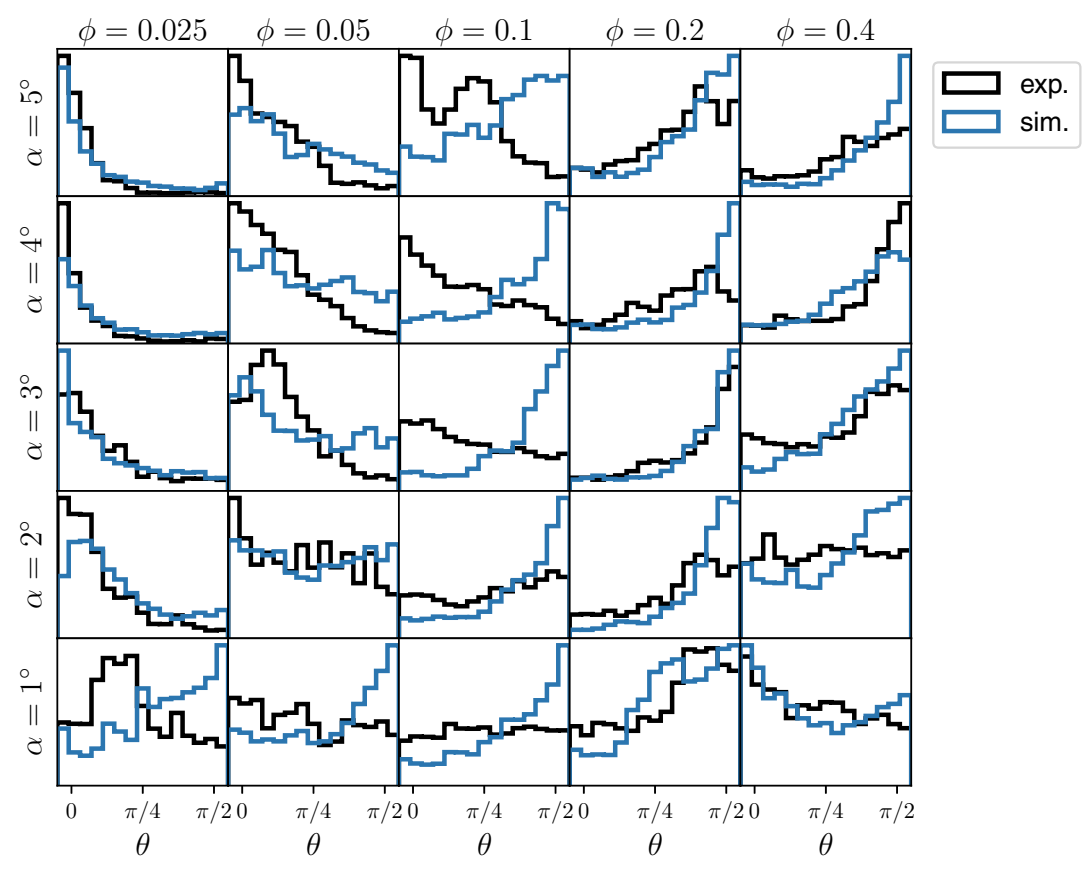

FIG. 7. Histograms of the alignment angle $\theta$ sample from the finger contour for each data point in the phase diagram in Fig. 6. The sample contains 10000 values of $\theta$ and the histograms consist of 15 equally spaced bins. Here $\theta=0$ and $\theta=\pi / 2$ correspond, respectively, to vertically and horizontally aligned finger structures.

to have its maximum at the boundaries of the domain of $\theta$, i.e., at $\theta=0$ for low $\eta$ and at $\theta=\pi / 2$ for the intermediate range of $\eta$.

Figure 8 nicely illustrates the transition from horizontally to vertically aligned fingers, but the transition from vertical to random alignment is harder to capture. For randomly aligned fingers we expect a uniform underlying distribution for $\theta$. The median should take values close to the center of the domain $\theta=\pi / 4$, whereas $\operatorname{SI}(p)$ could be any range determined by the fluctuation of the sample (the smallest interval containing a given fraction less than one of a distribution is nonunique for a uniform distribution). The figure also reveals that the simulated patterns undergo the transition from horizontal to vertical alignment at smaller values of $\eta$ than the experiments. This is likely due to inaccuracies in the specific set of parameters chosen for the simulation. A detailed analysis of how these parameters affect the transition is, however, outside the scope of this paper.

\section{APPLICATION: FLOW IN DIKES}

We now discuss the relevance of the studied system to magmatic flow during dike propagation. A (magmatic) dike is an approximately sheetlike body of magma, which has penetrated into a body of rock (host rock) at high angle to the bedding planes (e.g., sedimentary strata). Striking dike examples are found in the Inmar formation in the desert in southern Israel. There the magmatic rock (i.e., the solidified magma) of the dike has eroded away and both the erosion-resistant host, made of quartzitic sandstone, and outermost dikes' margins are exposed. The margins display a rich network of finger structures [37,38], similar to those described above. The fingers are identified as elongated grooves in the sandstone, separated by bulging ridges. The fingers are approximately $1-10 \mathrm{~cm}$ wide and $10-100$ $\mathrm{cm}$ long and the margins shows intermittent patches of finger alignment [Figs. 9(b) and 10(a)]. The walls are separated $\simeq 1 \mathrm{~m}$ apart, but mirror images of the finger structures on both walls suggest that 


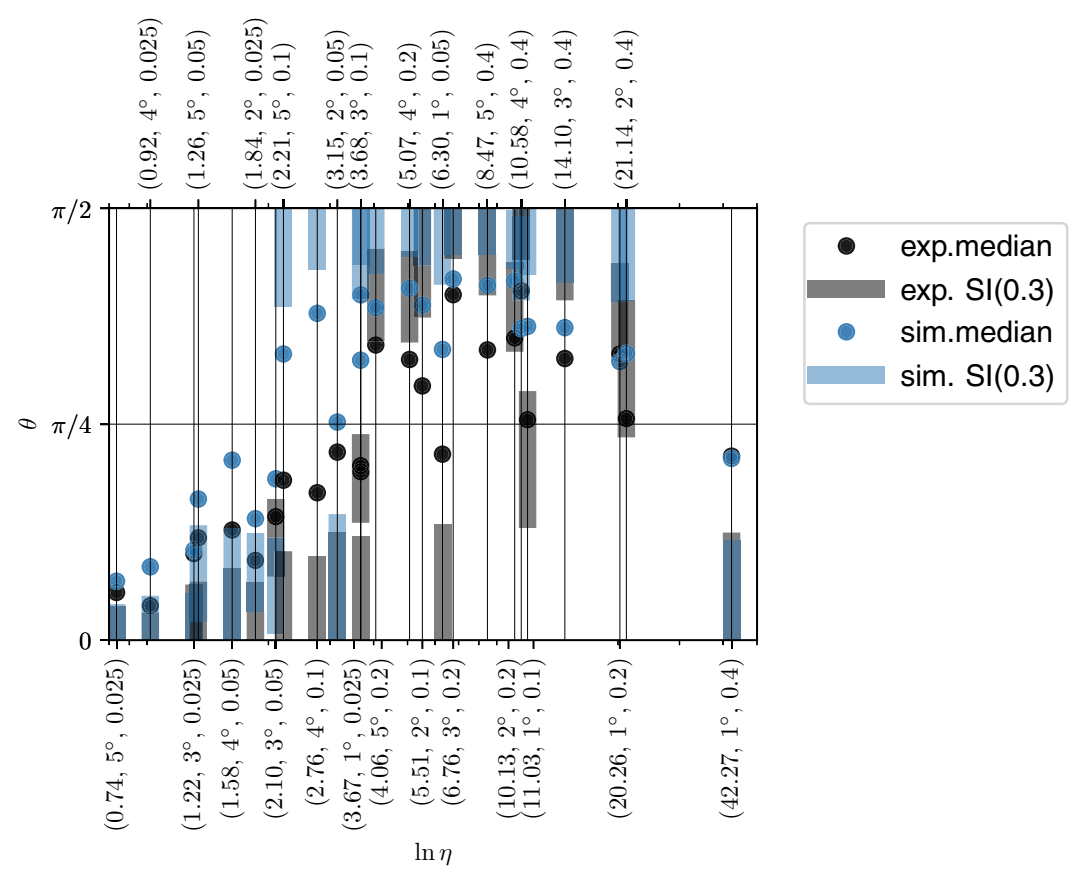

FIG. 8. Statistics of the samples in Fig. 7 plotted against $\ln \eta$. Circles correspond to sample medians and bars correspond to the smallest intervals of $\theta$ which contain $30 \%$ of the sample values, SI $(0.3)$. The triplets of numbers along the $\ln \eta$ axis indicate the values of $(\eta, \alpha, \phi)$ for each sample.

the structures were made during the initial stages of the dike formation, i.e., when the dike was thin and close to its propagating tip.

The finger formation in these dikes has previously been interpreted as viscous fingers due to the potential flow of a low-viscosity invading dike-related fluid into the higher-viscosity fluidized host

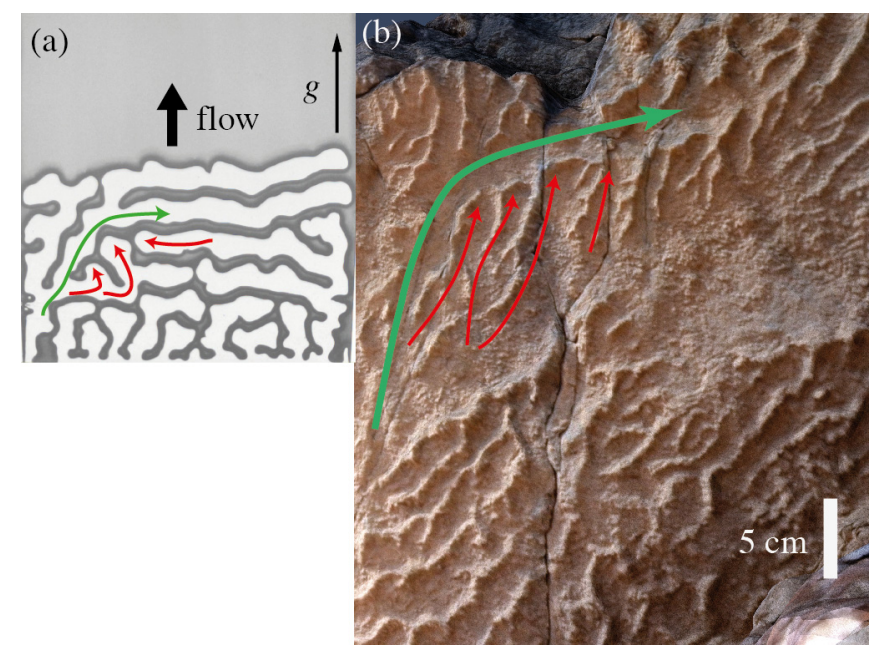

FIG. 9. Feature comparison 1 between (a) the experimental observations at $\phi=0.4$ and $\alpha=4^{\circ}$ and (b) the remaining structures on dike walls found in the Inmar formation. Fingers (red arrows) are being intercepted by a finger (green arrows) which grows perpendicular to the average flow direction. The gravitational pull is indicated by $g$. The scale bar in (b) applies to both experiment and the dike figure. 


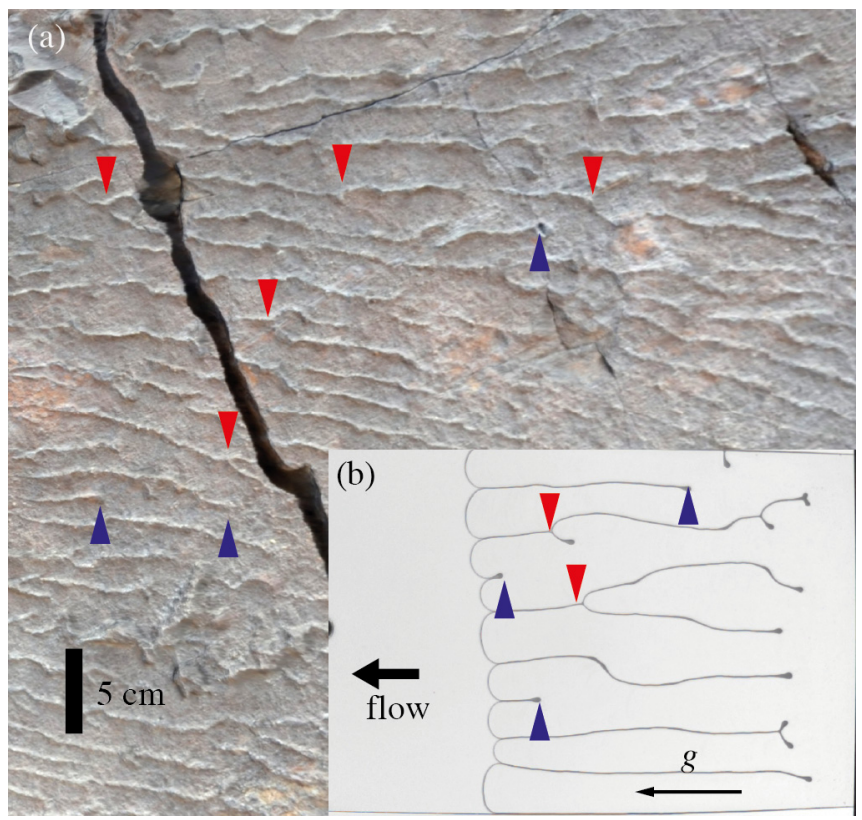

FIG. 10. Feature comparison 2 between (b) the experimental observations at $\phi=0.025$ and $\alpha=4^{\circ}$ and (a) the remaining structures on dike walls found in the Inmar formation. Aligned finger structures with tip splitting and termination are marked by blue and red triangles, respectively. The gravitational pull is indicated by $g$. The scale bar in (a) applies to both experiment and the dike figure.

rock [38]. Viscous fingers in porous media are, however, known to display fractal invasion patterns with no intrinsic length scale [8-10], whereas the fingers on the dike walls display a characteristic width. The similarity of these dike wall fingers to the aligned finger structures observed in our experimental setup suggests that the fingers are generated by intergranular friction between the quartz grains and accumulation of these grains onto stagnant fronts. The relevance of our system to the structure in the Inmar formation is further substantiated by the similarity in the features of the resulting pattern. In particular we observe similar tip splitting and termination properties (Fig. 10) and interception of fingers by a finger which grows perpendicular to the average flow direction (Fig. 9).

To test this hypothesis, we compare the geological observations to our model conditions. The ridges in the Inmar dikes are made of closely packed quartz grains (100-500 $\mu$ m diameter) cemented by iron oxides and kaolinite; this cement results from the weathering of the magmatic rock of the dike. This observation shows that (i) the quartz grains have resulted from local fluidization of the sandstone host rock and (ii) the quartz fragments have been locally mixed with the molten magma [38]. Such a quartz grain suspension is likely an equivalent to the bead-water-glycerol frictional fluid in our experiments. The similarity between the geological observations at the Inmar dikes and our system suggests that another fluid pushed away the quartz grain-magma mixture between the dike walls. The evidence of sandstone fluidization suggests that such fluid might be high-pressure overheated aqueous fluids hosted in the porous Inmar sandstone in the close vicinity of the dikes. Another type of magma could also behave as the invading fluid, as different magmas can behave as immiscible fluids during time scales which are relevant for this flow [42].

Steps between dike segments at the Inmar dikes suggest that the main propagation direction of the dikes was horizontal [38]. The fingers direction in the Inmar formation varies locally between vertical and horizontal, i.e., finger directions can be both perpendicular (Fig. 9) and parallel (Fig. 10), respectively, to the main dike propagation direction. Such variability is consistent with the distinct patterns displayed in the phase diagram of Fig. 6, which shows that the fingers can be either parallel 
or perpendicular to the main flow direction, depending on parameters such as gravity and the filling fraction of the frictional fluid. Such natural variability can be explained by the heterogeneous content of quartz grains mixed with the magma and varying opening of the dike. In addition, the direction of the gravitational effect on the fingers depends on the density contrast of the invading fluid to the quartz grain-magma mixture, which is unknown.

To summarize, the comparison between our physical results and the geological finger structures in dikes sheds light on the complex mechanisms at work during dike propagation and emplacement and especially the complex interactions between the intruding magma and its host rock.

\section{CONCLUSION}

We have described a type of pattern forming flow where grains are accumulated by a moving interface, which, when subject to a stabilizing potential, forms aligned finger structures. We identify the finger width by a work minimization principle and can estimate the alignment direction by the competition between frictional force fluctuations and the hydrostatic pressure. The dynamics is quasistatic; it depends on granular friction rather than viscosity. The patterning process seems to be independent of whether the invading fluid is a gas or a liquid, as long as the phases are immiscible. We can reproduce the finger behavior numerically by accounting for the hydrostatic pressure, grain accumulation, solid friction, and interfacial forces. As our model only contains geologically ubiquitous mechanisms, it may be relevant for a number of biphasic flow phenomena confined to planar fractures, in particular multiphase flow during dike formation that leave imprints of the finger formation as solidified granular residue on the dike walls.

\section{ACKNOWLEDGMENTS}

We are grateful to the late Henning Knudsen, who made important contributions to the understanding of frictional fingers. We thank Gidon Baer, Einat Aharonov, and Benjy Marks for discussions. J.A.E. acknowledges support from the Campus France Eiffel Grant and Unistra. This work was partly supported by the Research Council of Norway through the Center of Excellence funding scheme, Project No. 262644, and the NFR Project No. 200051/S60. B.S. acknowledges support from the EPSRC Grant No. EP/L013177/1 and Sêr Cymru National Research Network in Advanced Materials Grant No. NRN141. R.T., K.J.M., and E.F. acknowledge support from EU FP7 Grant No. 316889-ITN FlowTrans and from the LIA France-Norway D-FFRACT. R.T. also acknowledges additional support from UiO, Unistra, and the INSU ALEAS risk program.

[1] M. Sahimi, Flow and Transport in Porous Media and Fractured Rock (Wiley-VCH, Weinheim, 2011).

[2] M. C. Cross and P. C. Hohenberg, Pattern formation outside of equilibrium, Rev. Mod. Phys. 65, 851 (1993).

[3] P. G. Saffman and G. Taylor, The penetration of a fluid into a porous medium or Hele-Shaw cell containing a more viscous liquid, Proc. R. Soc. London Ser. A 245, 312 (1958).

[4] L. Paterson, Radial fingering in a Hele-Shaw cell, J. Fluid. Mech. 113, 513 (1981).

[5] D. Bensimon, L. P. Kadanoff, S. Liang, B. I. Shraiman, and C. Tang, Viscous flows in two dimensions, Rev. Mod. Phys. 58, 977 (1986).

[6] G. M. Homsy, Viscous fingering in porous media, Annu. Rev. Fluid Mech. 19, 271 (1987).

[7] L. Paterson, Diffusion-Limited Aggregation and Two-Fluid Displacements in Porous Media, Phys. Rev. Lett. 52, 1621 (1984).

[8] R. Lenormand and C. Zarcone, Invasion Percolation in an Etched Network: Measurement of a Fractal Dimension, Phys. Rev. Lett. 54, 2226 (1985). 
[9] K. J. Måløy, J. Feder, and T. Jøssang, Viscous Fingering Fractals in Porous Media, Phys. Rev. Lett. 55, 2688 (1985).

[10] J.-D. Chen and D. Wilkinson, Pore-Scale Viscous Fingering in Porous Media, Phys. Rev. Lett. 55, 1892 (1985).

[11] A. Ferrari, J. Jiminez-Martinez, T. Le Borgne, Y. Meheust, and I. Lunati, Challenges in modeling unstable two-phase flow experiments in porous micromodels, Water Resour. Res. 51, 1381 (2015).

[12] G. Taylor, The Instability of Liquid Surfaces when Accelerated in a Direction. Perpendicular to their Planes, Proc. R. London Ser. A 201, 192 (1958).

[13] J. J. Hidalgo, J. Fe, L. Cueto-Felgueroso, and R. Juanes, Scaling of Convective Mixing in Porous Media, Phys. Rev. Lett. 109, 264503 (2012).

[14] J.-H. Ching, P. Chen, and P. A. Tsai, Convective mixing in homogeneous porous media flow, Phys. Rev. Fluids 2, 014102 (2017).

[15] Y. Meheust, G. Løvoll, K. J. Måløy, and J. Schmittbuhl, Interface scaling in a two-dimensional porous medium under combined viscous, gravity, and capillary effects, Phys. Rev. E 66, 051603 (2002).

[16] F. Moebius and D. Or, Pore scale dynamics underlying the motion of drainage fronts in porous media, Water Resour. Res. 50, 8441 (2014).

[17] D. Amitrano and J. Schmittbuhl, Fracture roughness and gouge distribution of a granite shear band, J. Geophys. Res. 107, 2375 (2002).

[18] G. D. Nguyen and I. Einav, The energetics of cataclasis based on breakage mechanics, Pure Appl. Geophys. 166, 1693 (2009).

[19] H. Pinkerton, L. Wilson, and R. MacDonald, The transport and eruption of magma from volcanoes: A review, Contemp. Phys. 43, 197 (2002).

[20] J. Suckale, T. Keller, K. V. Cashman, and P.-O. Persson, Flow-to-fracture transition in a volcanic mush plug may govern normal eruptions at Stromboli, Geophys. Res. Lett. 43, 12071 (2016).

[21] K. Cashman, R. S. J. Sparks, and J. D. Blundy, Vertically extensive and unstable magmatic systems: A unified view of igneous processes, Science 355, 6331 (2017).

[22] B. Sandnes, E. G. Flekkøy, H. A. Knudsen, K. J. Måløy, and H. See, Patterns and flow in frictional fluid dynamics, Nat. Commun. 2, 288 (2011).

[23] C. Chevalier, A. Lindner, M. Leroux, and E. Clement, Morphodynamics during air injection into a confined granular suspension, J. Non-Newton. Fluid 158, 63 (2009).

[24] H. Shin and J. C. Santamarina, Fluid-driven fractures in uncemented sediments: Underlying particle-level processes, Earth Planet. Sci. Lett. 299, 180 (2010).

[25] G. Varas, V. Vidal, and J.-C. Geminard, Venting dynamics of an immersed granular layer, Phys. Rev. E 83, 011302 (2011).

[26] R. Holtzman, M. L. Szulczewski, and R. Juanes, Capillary Fracturing in Granular Media, Phys. Rev. Lett. 108, 264504 (2012).

[27] J. Oppenheimer, A. C. Rust, K. V. Cashman, and B. Sandnes, Gas migration regimes and outgassing in particle-rich suspensions, Front. Phys. 3, 60 (2015).

[28] Ø. Johnsen, C. Chevalier, A. Lindner, R. Toussaint, E. Clément, K. J. Måløy, E. G. Flekkøy, and J. Schmittbuhl, Decompaction and fluidization of a saturated and confined granular medium by injection of a viscous liquid or gas, Phys. Rev. E 78, 051302 (2008).

[29] H. A. Knudsen, B. Sandnes, E. G. Flekkøy, and K. J. Måløy, Granular labyrinth structures in confined geometries, Phys. Rev. E 77, 021301 (2008).

[30] J. A. Eriksen, B. Marks, B. Sandnes, and R. Toussaint, Bubbles breaking the wall: Two-dimensional stress and stability analysis, Phys. Rev. E 91, 052204 (2015).

[31] B. Sandnes, H. A. Knudsen, K. J. Måløy, and E. G. Flekkøy, Labyrinth Patterns in Confined Granular-Fluid Systems, Phys. Rev. Lett. 99, 038001 (2007).

[32] J. A. Eriksen, R. Toussaint, K. J. Måløy, E. Flekkøy, and B. Sandnes, Numerical approach to frictional fingers, Phys. Rev. E 92, 032203 (2015).

[33] J. D. Clemens and C. K. Mawer, Granitic magma transport by fracture propagation, Tectonophysics 204, 339 (1992). 
[34] T. J. Wright, F. Sigmundsson, C. Pagli, M. Belachew, I. J. Hamling, B. Brandsdóttir, D. Keir, R. Pedersen, A. Ayele, C. Ebinger, P. Einarsson, E. Lewi, and E. Calais, Geophysical constraints on the dynamics of spreading centres from rifting episodes on land, Nat. Geosci. 5, 242 (2012).

[35] F. Sigmundsson et al., Segmented lateral dyke growth in a rifting event at Bárðarbunga volcanic system, Iceland, Nature (London) 517, 191 (2015).

[36] E. Rivalta, B. Taisne, A. P. Bunger, and R. F. Katz, A review of mechanical models of dike propagation: Schools of thought, results and future directions, Tectonophysics 638, 1 (2015).

[37] G. Baer and Z. Reches, Flow patterns of magma in dikes, Makhtesh Ramon, Israel, Geology 15, 569 (1987).

[38] G. Baer, Mechanisms of dike propagation in layered rocks and in massive, porous sedimentary rocks, J. Geophys. Res. Solid Earth 96, 11911 (1991).

[39] See Supplemental Material at http://link.aps.org/supplemental/10.1103/PhysRevFluids.3.013801 for movies of experiments and simulations of vertically aligned fingers (SM video 1) and horizontally aligned fingers (SM video 2).

[40] U. Thiele, I. Vancea, A. J. Archer, M. J. Robbins, L. Frastia, A. Stannard, E. Pauliac-Vaujour, C. P. Martin, M. O. Blunt, and P. J. Moriarty, Modelling approaches to the dewetting of evaporating thin films of nanoparticle suspensions, J. Phys.: Condens. Matter 21, 264016 (2009).

[41] G. Dumazer, B. Sandnes, M. Ayaz, K. J. Måløy, and E. G. Flekkøy, Frictional Fluid Dynamics and Plug Formation in Multiphase Millifluidic Flow, Phys. Rev. Lett. 117, 028002 (2016).

[42] G. Charreteur and C. Tegner, Magmatic emulsion texture formed by mixing during extrusion, Rauðafell composite complex, Breiðdalur volcano, eastern Iceland, Bull. Volcanol. 75, 721 (2013). 